Supplement of Atmos. Chem. Phys., 19, 14107-14117, 2019

https://doi.org/10.5194/acp-19-14107-2019-supplement

(c) Author(s) 2019. This work is distributed under

the Creative Commons Attribution 4.0 License.

(c) (i)

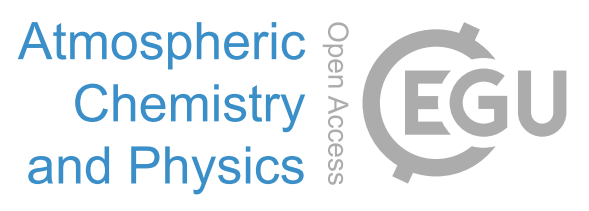

Supplement of

\title{
Occurrence and source apportionment of perfluoroalkyl acids (PFAAs) in the atmosphere in China
}

Deming Han et al.

Correspondence to: Jinping Cheng (jpcheng@sjtu.edu.cn)

The copyright of individual parts of the supplement might differ from the CC BY 4.0 License. 


\section{Supplementary material}

\section{CONTENT}

Table S1. Physical and chemical properties of target PFAAs compounds. 2

Table S2. The geographic information and annual temperature in different sampling sites of atmospheric PFAAs....3

Table S3. MS parameters, MDLs, LODs, LOQs values and recovery rates for individual compounds of PFAAs...... 5

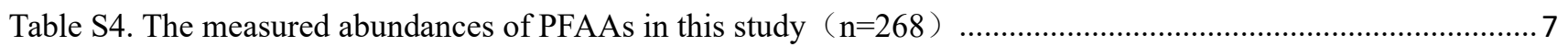

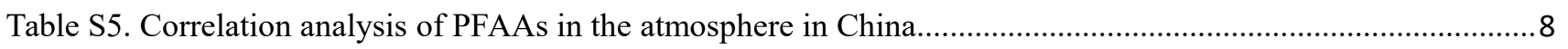

Figure S1. Spatial distributions of 23 sampling sites of atmospheric PFAAs in China ..........................................9

Figure S2. Temporal variations of PFAAs concentrations in selected four typical sites........................................... 10

Figure S3. The spatial distributions of fluoride related products manufacturers in China....................................... 11

Figure S4. The spatial distributions of fluoride related products manufacturers in Zhejiang site ...........................12

Figure S5. The backward trajectories of air mass extracted by Hysplit trajectory model......................................... 14

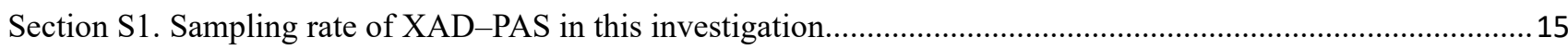

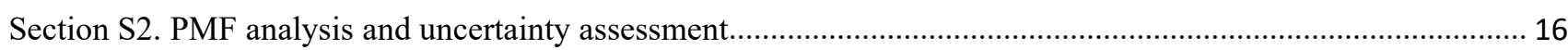

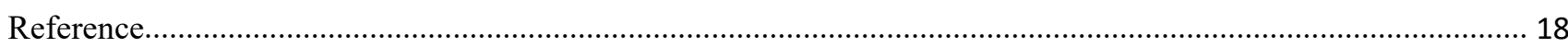

NINETEEN pages: FIVE tables and FIVE figures, TWO sections,. 
Table S1. Physical and chemical properties of target PFAAs compounds

\begin{tabular}{|c|c|c|c|c|c|c|}
\hline Component & Abbreviation & Molecular & Molecular & Bio-concentr & $\log K_{\mathrm{OW}}{ }^{\mathrm{b}}$ & $\mathrm{P}_{\mathrm{L}}^{\mathrm{o}}(\mathrm{mmHg})^{\mathrm{c}}$ \\
\hline & & structure & weight & ation factor ${ }^{a}$ & & \\
\hline \multicolumn{7}{|c|}{ Perfluoroalkane carboxylic acids (PFCAs) } \\
\hline Perfluoropentanoic acid & PFPeA & C4F9COOH & 263.98 & 1.00 & 5.29 & $7.9 \pm 0.4$ \\
\hline Perfluorohexanoic acid & PFHxA & $\mathrm{C} 5 \mathrm{~F} 11 \mathrm{COOH}$ & 313.98 & 1.00 & 5.97 & $3.1 \pm 0.5$ \\
\hline Perfluoroheptanoic acid & PFHpA & $\mathrm{C} 6 \mathrm{~F} 13 \mathrm{COOH}$ & 363.97 & 1.00 & 6.86 & $0.5 \pm 0.6$ \\
\hline Perfluorooctanoic acid & PFOA & $\mathrm{C} 7 \mathrm{~F} 15 \mathrm{COOH}$ & 413.97 & 1.90 & 7.75 & $0.3 \pm 0.7$ \\
\hline Perfluorononanoic acid & PFNA & $\mathrm{C} 8 \mathrm{~F} 17 \mathrm{COOH}$ & 463.97 & 11.26 & 8.64 & $0.2 \pm 0.8$ \\
\hline Perfluorodecanoic acid & PFDA & C9F19COOH & 513.96 & 44.30 & 9.53 & $0.0 \pm 0.9$ \\
\hline Perfluoroundecanoic acid & PFUdA & $\mathrm{C} 10 \mathrm{~F} 21 \mathrm{COOH}$ & 563.96 & 128.19 & 10.42 & $0.0 \pm 0.9$ \\
\hline Perfluorododecanoic acid & PFDoA & $\mathrm{C} 11 \mathrm{~F} 23 \mathrm{COOH}$ & 613.95 & 235.68 & 11.31 & $0.0 \pm 1.0$ \\
\hline Perfluorotridecanoic acid & PFTrDA & $\mathrm{C} 12 \mathrm{~F} 25 \mathrm{COOH}$ & 663.95 & 474.19 & 12.19 & $0.0 \pm 1.1$ \\
\hline Perfluorotetradecanoic acid & PFTeDA & $\mathrm{C} 13 \mathrm{~F} 27 \mathrm{COOH}$ & 713.95 & 1903.40 & 13.08 & $0.0 \pm 1.2$ \\
\hline
\end{tabular}

Perfluoroalkane sulfonic acids (PFSAs)

\begin{tabular}{llccccc}
\hline Perfluorobutane sulfonic acid & PFBS & C4F9SO3H & 299.98 & 1.00 & 3.68 & $/ \mathrm{d}$ \\
Perfluorohexane sulfonic acid & PFHxS & C6F13SO3H & 399.97 & 1.00 & 5.25 & $/$ \\
Perfluorooctane sulfonic acid & PFOS & C8F17SO3H & 499.97 & 1.00 & 7.03 & $/$ \\
\hline
\end{tabular}

\footnotetext{
a: Predicted data are generated using the Advanced Chemistry Development, Inc. (Canada), cited from (Yu, Liu et al. 2018);

b: Predicted octanol-water partitioning coefficients from individual PFAAs structure, cited from (Buck, Franklin et al. 2011, Yu, Liu et al. 2018);

c: Predicted pure compound vapor pressure, unit of mmHg at $298 \mathrm{~K}$, cited from (Buck, Franklin et al. 2011, Yu, Liu et al. 2018);

d. “/” means lack of related data.
} 
Table S2. The geographic information and annual temperature in different sampling sites of atmospheric PFAAs

\begin{tabular}{|c|c|c|c|c|c|c|c|c|c|c|}
\hline I.D. & Region & & Province & Type & Location & Elevati & Monthly & Domestic & Resident & Crude plastic \\
\hline & & & & & & on $(\mathrm{m})$ & temperature $\left({ }^{\circ} \mathrm{C}\right)^{\mathrm{a}}$ & Product $\left(10^{8} \mathrm{RMB}\right)^{\mathrm{b}}$ & population $\left(10^{4}\right)^{b}$ & $\left(10^{4} \text { tons }\right)^{\mathrm{b}}$ \\
\hline 1 & Northern & of & Beijing & Urban & Haidian District & 31 & $-5-24$ & 127.75 & 2171 & 28014.94 \\
\hline 2 & China, NC & & Tianjin & Urban & Jinnan District & 3.3 & $-4-25$ & 332.42 & 1557 & 18549.19 \\
\hline 3 & & & Shanxi & Rural & Linshui County, Jincheng city & 376 & $-11-17$ & 79.47 & 3702 & 15528.42 \\
\hline 4 & Eastern & of & Shanghai & Urban & Minhang District & 4.5 & $5-28$ & 364.04 & 2418 & 30632.99 \\
\hline 5 & China, EC & & Zhejiang & Rural & Yinzhou District, Ningbo City & 4 & $4-23$ & 896.29 & 5657 & 51768.26 \\
\hline 6 & & & Jiangsu & Urban & Changzhou City & 5 & $2-26$ & 1175.39 & 8209 & 85869.76 \\
\hline 7 & & & Anhui & Urban & Yinquan District, Fuyang City & 30 & $2-27$ & 137.35 & 6225 & 27018 \\
\hline 8 & & & Fujian & Urban & Huian Country, Quanzhou City & 30 & $12-26$ & 235.74 & 3911 & 32182.09 \\
\hline 10 & & & Jiangxi & Urban & Jiujiang City & 32.2 & $4-26$ & 25.46 & 4622 & 20006.31 \\
\hline 9 & & & Shandong & Urban & Laishan District, Yantai City & 47 & $-1-24$ & 710.42 & 10006 & 72634.15 \\
\hline 11 & Southern & of & Guangdogn & Urban & Nanshan District, Shenzhen City & 7 & $15-26$ & 695.31 & 11169 & 89705.26 \\
\hline
\end{tabular}




\begin{tabular}{|c|c|c|c|c|c|c|c|c|c|}
\hline 12 & China, SC & Hainan & Urban & Meilan District, Haikou City & 12 & $18-26$ & 19.67 & 926 & 4462.54 \\
\hline 13 & Central & Hubei & Urban & Yunxi District, Shiyan City & 437 & $1-24$ & 191.86 & 5902 & 35478.09 \\
\hline 14 & China, CC & Henan & Urban & Gaoxin District, Zhenzhou City & 110 & $-2-26$ & 232.47 & 9559 & 44552.83 \\
\hline 15 & & Hunan & Urban & Huaxin District, Hengyang City & 103 & $7-27$ & 48.4 & 6860 & 33902.96 \\
\hline 16 & Northwestern of & Xinjiang & Urban & Tacheng City & 427 & $-14-18$ & 621.72 & 2445 & 10881.96 \\
\hline 17 & China, NW & Shaanxi & Urban & Beilin District, Xi'an City & 397 & $-1-24$ & 478.63 & 3835 & 21898.81 \\
\hline 18 & & Gansu & Urban & Chengguang District, Lanzhou City & 1517 & $-7-19$ & 121.57 & 2626 & 7459.9 \\
\hline 19 & Southwestern of & Sichuan & Urban & Shuangliu District, Chengdu City & 506 & $4-23$ & 214.94 & 3789 & 15901.68 \\
\hline 20 & China, SW & Yunnan & Urban & Lanchang Country, Puer City & 1950 & $3-19$ & 319.76 & 4369 & 23409.24 \\
\hline 21 & & Guizhou & Urban & Xinren Country, Qiandongnan City & 1379 & $6-22$ & 127.75 & 2171 & 28014.94 \\
\hline 22 & Northeastern of & Heilongjiang & Urban & Beilin District, Suihua City & 172 & $-22-19$ & 332.42 & 1557 & 18549.19 \\
\hline 23 & China, NE & Liaoning & Rural & Neizhou Country,Huludao City & 118 & $-12-21$ & 79.47 & 3702 & 15528.42 \\
\hline
\end{tabular}

2 a: Meteorological data originated from China Meteorological Administration, http://www.cma.gov.cn/;

3 b: Data originated from China Statistic Yearbook 2018, National Bureau of Statistics China, http://www.stats.gov.cn/tjsj/ndsj/; 
Table S3. MS parameters, MDLs, LODs, LOQs values, recovery rates and blank values for individual compounds of PFAAs

\begin{tabular}{|c|c|c|c|c|c|c|c|c|c|c|c|c|}
\hline Analogues & Parent & Daughter & Declustering & Collision & Retention & MDLs & LODs & LOQs & Recovery & Filed bank & Laboratory & Internal Standards \\
\hline & ions $(\mathrm{m} / \mathrm{z})$ & ion $(\mathrm{m} / \mathrm{z})$ & potential $(\mathrm{V})^{\mathrm{a}}$ & energy $(\mathrm{eV})^{\mathrm{b}}$ & time $(\mathrm{s})$ & $\left(\mathrm{pg} / \mathrm{m}^{3}\right)$ & $\left(\mathrm{pg} / \mathrm{m}^{3}\right)$ & $\left(\mathrm{pg} / \mathrm{m}^{3}\right)$ & rate $(\%)$ & $\left(\mathrm{pg} / \mathrm{m}^{3}\right)$ & blank (pg/m³) & \\
\hline \multicolumn{13}{|l|}{ PFCAs } \\
\hline PFPeA & 263 & 219 & -40 & -34 & 3.16 & 0.41 & 0.31 & 1.05 & $96 \pm 17$ & $0.41 \pm 0.14$ & $0.22 \pm 0.17$ & $1,2-{ }^{13} \mathrm{C}_{2}-\mathrm{PFHxA}$ \\
\hline PFHxA & 313 & 269 & -35 & -36 & 3.42 & 0.18 & 0.14 & 0.47 & $108 \pm 22$ & $0.48 \pm 0.06$ & $0.37 \pm 0.39$ & $1,2-{ }^{13} \mathrm{C}_{2}-\mathrm{PFHxA}$ \\
\hline PFHpA & 363 & $319 \rightarrow 169$ & -55 & -28 & 3.70 & 0.22 & 0.16 & 0.55 & $93 \pm 16$ & $0.62 \pm 0.07$ & $0.22 \pm 0.32$ & $1,2,3,4-{ }^{13} \mathrm{C}_{4}-\mathrm{PFOA}$ \\
\hline PFOA & 413 & $369 \rightarrow 169$ & -45 & -39 & 3.99 & 0.33 & 0.26 & 0.87 & $91 \pm 13$ & $0.93 \pm 0.11$ & $0.41 \pm 0.29$ & $1,2,3,4-{ }^{13} \mathrm{C}_{4}-\mathrm{PFOA}$ \\
\hline PFNA & 463 & $419 \rightarrow 219$ & -40 & -44 & 4.32 & 0.61 & 0.46 & 1.53 & $89 \pm 17$ & $0.57 \pm 0.20$ & $0.20 \pm 0.25$ & $1,2,3,4,5-{ }^{13} \mathrm{C}_{5}-\mathrm{PFNA}$ \\
\hline PFDA & 513 & $469 \rightarrow 219$ & -50 & -47 & 4.67 & 0.56 & 0.42 & 1.39 & $93 \pm 11$ & $0.35 \pm 0.19$ & $0.28 \pm 0.22$ & $1,2-{ }^{13} \mathrm{C}_{2}-\mathrm{PFDA}$ \\
\hline PFUdA & 563 & $519 \rightarrow 269$ & -45 & -61 & 5.02 & 0.28 & 0.21 & 0.70 & $88 \pm 16$ & $0.31 \pm 0.09$ & $0.31 \pm 0.13$ & $1,2-{ }^{13} \mathrm{C}_{2}-\mathrm{PFUdA}$ \\
\hline PFDoA & 613 & $569 \rightarrow 169$ & -45 & -65 & 5.35 & 0.28 & 0.21 & 0.70 & $94 \pm 18$ & $0.44 \pm 0.09$ & $0.15 \pm 0.18$ & $1,2-{ }^{13} \mathrm{C}_{2}-\mathrm{PFDoA}$ \\
\hline PFTrDA & 663 & $619 \rightarrow 169$ & -50 & -59 & 5.64 & 0.34 & 0.26 & 0.87 & $102 \pm 17$ & $0.09 \pm 0.11$ & $0.05 \pm 0.11$ & $1,2-{ }^{13} \mathrm{C}_{2}-\mathrm{PFDoA}$ \\
\hline PFTeDA & 713 & $669 \rightarrow 169$ & -65 & -57 & 5.94 & 0.14 & 0.31 & 1.03 & $97 \pm 21$ & $0.12 \pm 0.14$ & $0.06 \pm 0.13$ & $1,2-{ }^{13} \mathrm{C}_{2}-\mathrm{PFDoA}$ \\
\hline PFSAs & & & & & & & & & & & & \\
\hline
\end{tabular}




\begin{tabular}{|c|c|c|c|c|c|c|c|c|c|c|c|c|}
\hline PFBS & 299 & $80 \rightarrow 99$ & -45 & -64 & 3.19 & 0.25 & 0.20 & 0.66 & $81 \pm 25$ & $0.11 \pm 0.08$ & $0.27 \pm 0.46$ & ${ }^{18} \mathrm{O}_{2}-\mathrm{PFHxS}$ \\
\hline PFHxS & 399 & $80 \rightarrow 99$ & -55 & -87 & 3.70 & 0.16 & 0.12 & 0.40 & $86 \pm 13$ & $0.16 \pm 0.05$ & $0.42 \pm 0.27$ & ${ }^{18} \mathrm{O}_{2}-\mathrm{PFHxS}$ \\
\hline PFOS & 499 & $80 \rightarrow 99$ & -55 & -98 & 4.31 & 0.24 & 0.19 & 0.63 & $95 \pm 15$ & $0.75 \pm 0.08$ & $0.54 \pm 0.61$ & $1,2,3,4-{ }^{13} \mathrm{C}_{4}-\mathrm{PFOS}$ \\
\hline \multicolumn{13}{|l|}{ Internal Standards } \\
\hline $1,2-{ }^{13} \mathrm{C}_{2}-\mathrm{PFHxA}$ & 315 & 270 & -75 & -41 & 3.40 & / & / & / & l & l & l & / \\
\hline $1,2,3,4-{ }^{13} \mathrm{C}_{4}-\mathrm{PFOA}$ & 417 & 372 & -40 & -41 & 3.99 & / & l & l & l & / & l & l \\
\hline $1,2,3,4,5-{ }^{13} \mathrm{C}_{5}-\mathrm{PFNA}$ & 468 & 423 & -84 & -52 & 4.34 & / & I & I & / & I & l & / \\
\hline $1,2-{ }^{13} \mathrm{C}_{2}-\mathrm{PFDA}$ & 515 & 470 & -87 & -51 & 4.69 & / & l & / & / & / & l & / \\
\hline $1,2-{ }^{13} \mathrm{C}_{2}-\mathrm{PFUdA}$ & 565 & 520 & -79 & -61 & 5.02 & l & l & I & l & l & l & / \\
\hline $1,2-{ }^{13} \mathrm{C}_{2}-\mathrm{PFDoA}$ & 615 & 570 & -66 & -55 & 5.35 & / & l & / & / & / & l & l \\
\hline${ }^{18} \mathrm{O}_{2}-\mathrm{PFHxS}$ & 403 & 103 & -55 & 97 & 3.72 & / & / & / & / & / & l & / \\
\hline $1,2,3,4-{ }^{13} \mathrm{C}_{4}-\mathrm{PFOS}$ & 503 & 80 & -80 & 97 & 4.31 & / & l & l & l & / & l & l \\
\hline
\end{tabular}

a. cited from Karásková et al., 2018.

b: cited from Karásková et al., 2018 and Liu et al., 2015. 
Table S4. The measured abundances of PFAAs in this study $(\mathbf{n}=\mathbf{2 6 8})$

\begin{tabular}{|c|c|c|c|c|c|c|}
\hline Analogues & Detection & Average value & Standard deviation & Minimum value & Maximum value & Median value \\
\hline & frequency $(\%)$ & $\left(\mathrm{pg} / \mathrm{m}^{3}\right)$ & $\left(\mathrm{pg} / \mathrm{m}^{3}\right)$ & $\left(\mathrm{pg} / \mathrm{m}^{3}\right)$ & $\left(\mathrm{pg} / \mathrm{m}^{3}\right)$ & $\left(\mathrm{pg} / \mathrm{m}^{3}\right)$ \\
\hline \multicolumn{7}{|l|}{ PFCAs } \\
\hline PFPeA & 84.8 & 4.96 & 4.77 & $\mathrm{BDL}$ & 35.2 & 3.55 \\
\hline PFHxA & 92.1 & 5.36 & 7.17 & BDL & 79.7 & 3.73 \\
\hline PFHpA & 94.7 & 3.42 & 3.71 & $\mathrm{BDL}$ & 28.9 & 2.39 \\
\hline PFOA & 100 & 8.19 & 8.03 & 0.36 & 70.4 & 6.24 \\
\hline PFNA & 96.6 & 3.07 & 2.77 & BDL & 22.7 & 2.52 \\
\hline PFDA & 96.2 & 4.13 & 3.74 & $\mathrm{BDL}$ & 30.5 & 3.36 \\
\hline PFUdA & 75.6 & 1.24 & 1.32 & BDL & 6.72 & 0.86 \\
\hline PFDoA & 63.5 & 0.56 & 0.50 & BDL & 3.18 & 0.45 \\
\hline PFTrDA & 37.3 & 0.58 & 0.56 & BDL & 3.57 & 0.47 \\
\hline PFTeDA & 41.7 & 0.19 & 0.25 & BDL & 2.25 & 0.11 \\
\hline \multicolumn{7}{|l|}{ PFSAs } \\
\hline PFBS & 62.2 & 1.96 & 1.85 & BDL & 9.39 & 1.37 \\
\hline PFHxS & 71.6 & 0.99 & 1.38 & $\mathrm{BDL}$ & 13.2 & 0.56 \\
\hline PFOS & 100 & 5.20 & 4.30 & 0.34 & 25.5 & 3.87 \\
\hline
\end{tabular}

BDL: below detection limit. 
Table S5. Correlation analysis of PFAAs in the atmosphere in China

\begin{tabular}{|c|c|c|c|c|c|c|c|c|c|c|c|c|}
\hline & PFPeA & PFHxA & РFHрA & PFOA & PFNA & PFDA & PFUdA & PFDoA & PFTrDA & PFTeDA & PFBS & PFHxS \\
\hline PFHxA & $0.70^{* *}$ & & & & & & & & & & & \\
\hline PFHpA & 0.12 & $0.31^{*}$ & & & & & & & & & & \\
\hline PFOA & $0.69^{* * *}$ & $0.77^{\text {** }}$ & $0.68^{* *}$ & & & & & & & & & \\
\hline PFNA & $0.66^{* *}$ & $0.66^{* *}$ & $0.65^{* *}$ & $0.70^{* *}$ & & & & & & & & \\
\hline PFDA & $0.54^{* *}$ & $0.67^{\text {** }}$ & $0.72^{* *}$ & $0.84^{* *}$ & $0.61^{* *}$ & & & & & & & \\
\hline PFUdA & 0.16 & 0.32 & 0.15 & 0.2 & 0.14 & 0.23 & & & & & & \\
\hline PFDoA & $0.39^{*}$ & 0.33 & 0.27 & 0.38 & 0.31 & 0.32 & $0.61^{* *}$ & & & & & \\
\hline PFTrDA & $0.53^{*}$ & $0.48^{* *}$ & 0.3 & 0.42 & 0.44 & $0.51^{* *}$ & $0.65^{* *}$ & $0.62^{* * *}$ & & & & \\
\hline PFTeDA & 0.21 & 0.4 & $0.39^{*}$ & $0.36^{*}$ & 0.27 & 0.39 & $0.72^{* *}$ & $0.59^{* *}$ & $0.79^{* *}$ & & & \\
\hline PFBS & $0.68^{* * *}$ & 0.26 & 0.15 & 0.26 & 0.15 & 0.39 & 0.14 & 0.23 & 0.28 & 0.18 & & \\
\hline PFHxS & $0.57^{*}$ & $0.69^{* *}$ & 0.27 & $0.42^{*}$ & $0.57^{* *}$ & $0.64^{* *}$ & 0.3 & $0.43^{*}$ & $0.54^{*}$ & $0.38^{*}$ & 0.28 & \\
\hline PFOS & $0.69^{* *}$ & $0.42^{*}$ & 0.32 & 0.33 & 0.36 & $0.37^{*}$ & 0.25 & $0.41^{*}$ & $0.46^{*}$ & 0.38 & $0.63^{* *}$ & $0.40^{*}$ \\
\hline
\end{tabular}

\footnotetext{
*: represent $\mathrm{p}<0.05$

**: represent $\mathrm{p}<0.01$
} 


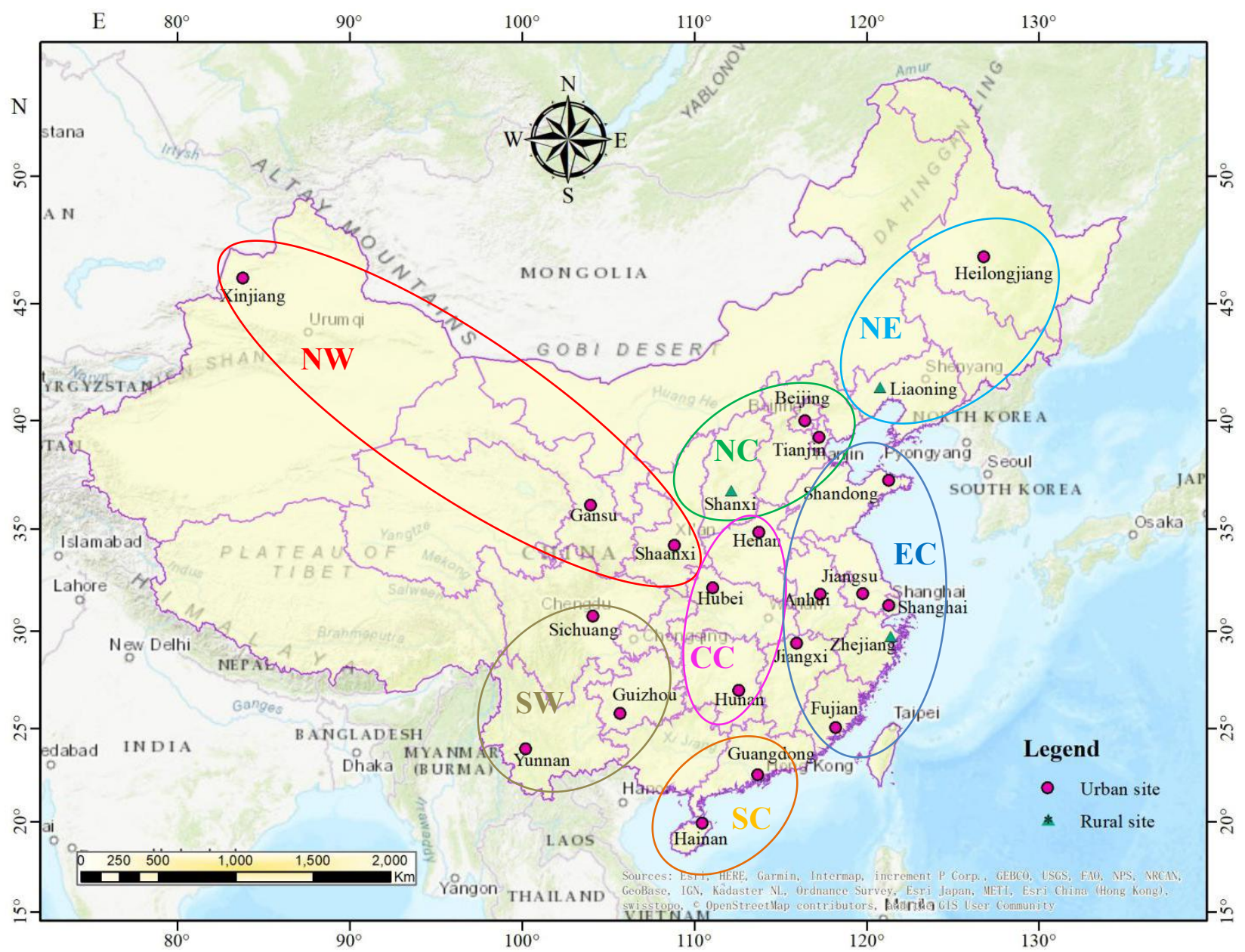

Figure S1. Spatial distributions of 23 sampling sites of atmospheric PFAAs in China (including 20 urban sites, red circles; and three rural site, green triangles). 

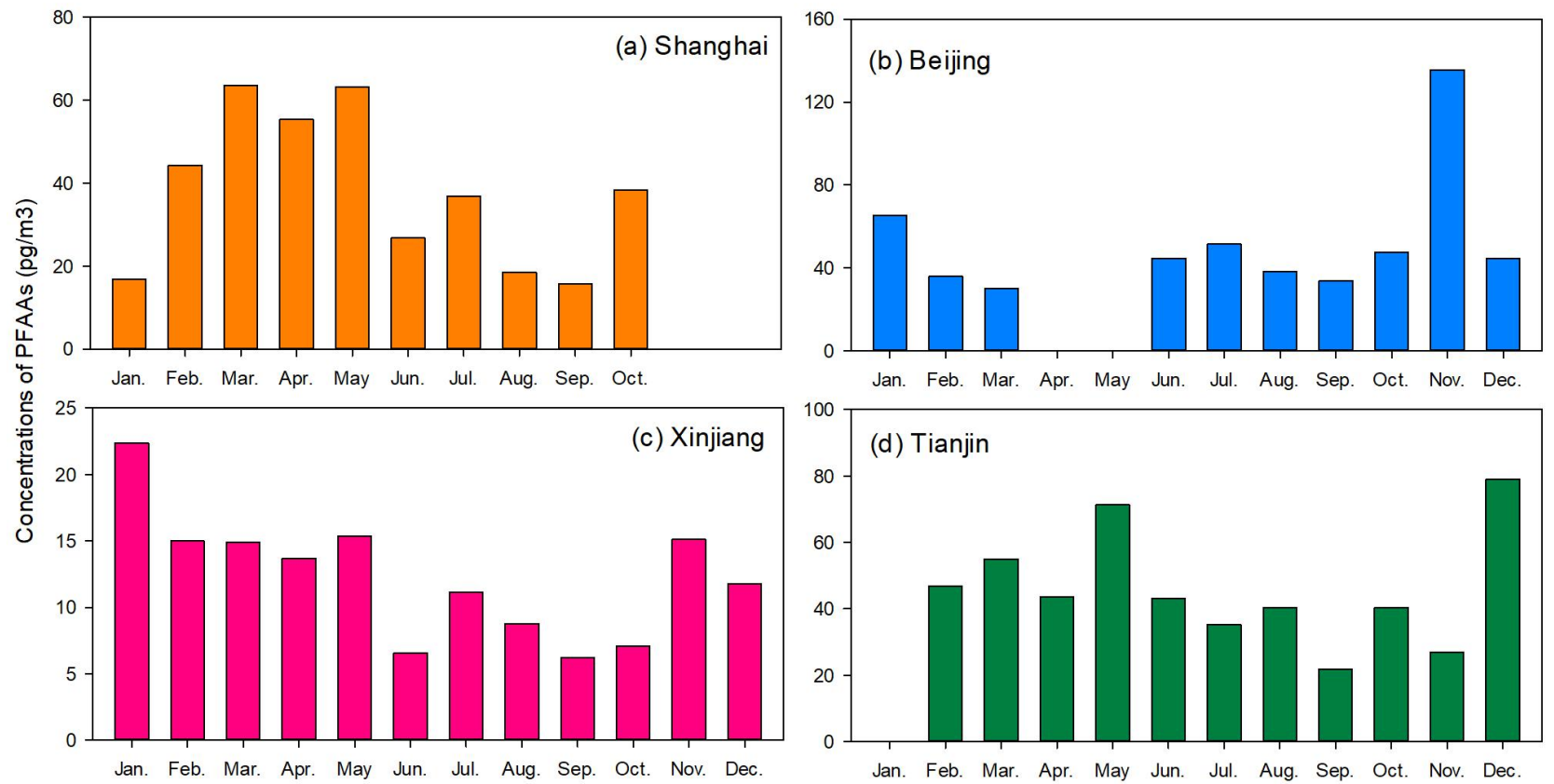

Sampling Time (month)

Figure S2. Temporal variations of PFAAs concentrations in selected four typical sites: Shanghai, Beijing, Xinjiang and Tianjin. 


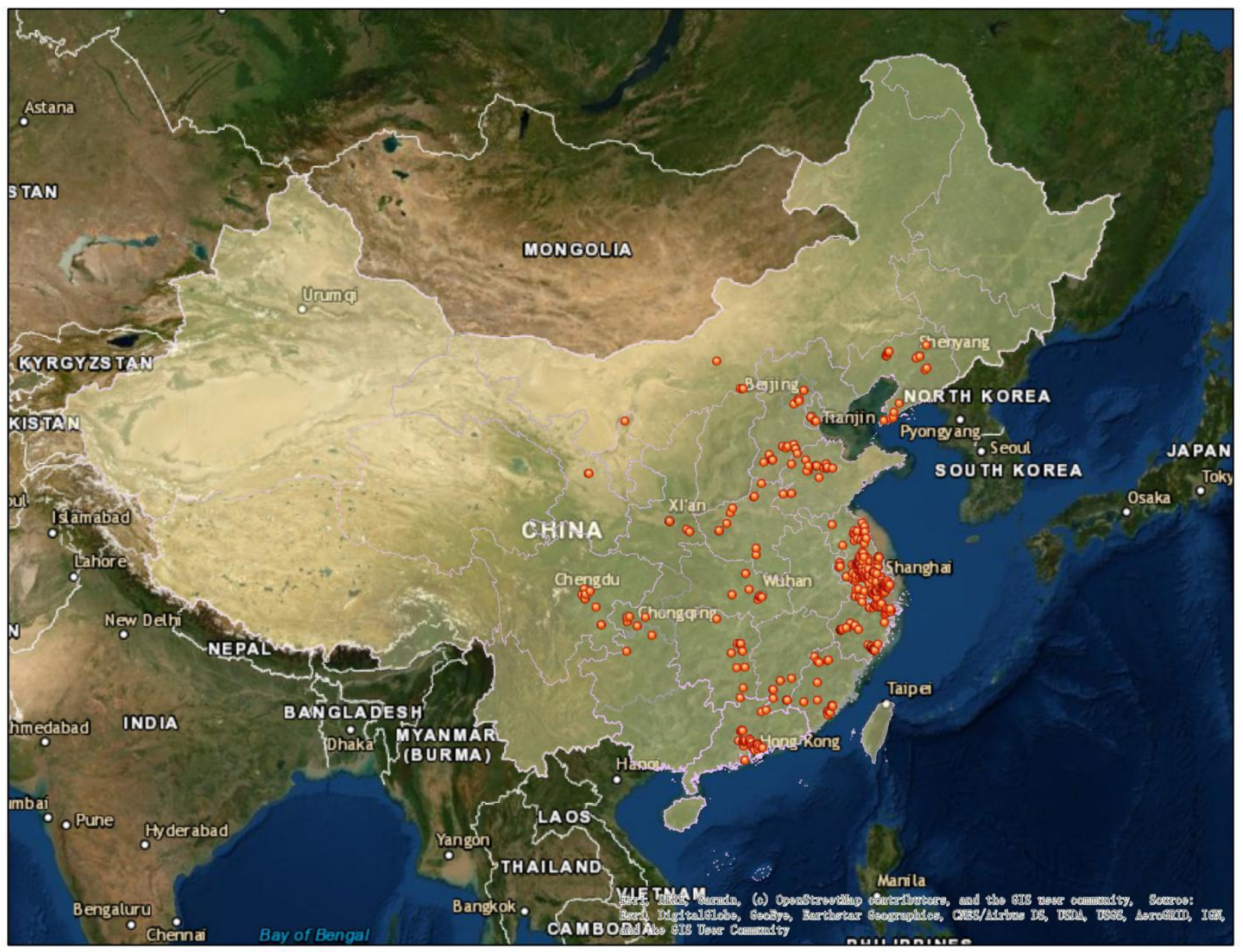

Figure S3. The spatial distributions of fluoride related products manufacturers in China and the different geographical conditions (note that the fluoride related manufacturers including textiles, crude plastic, paint coating, packaging materials, while part of fluoride related industries were not included in this figure) 


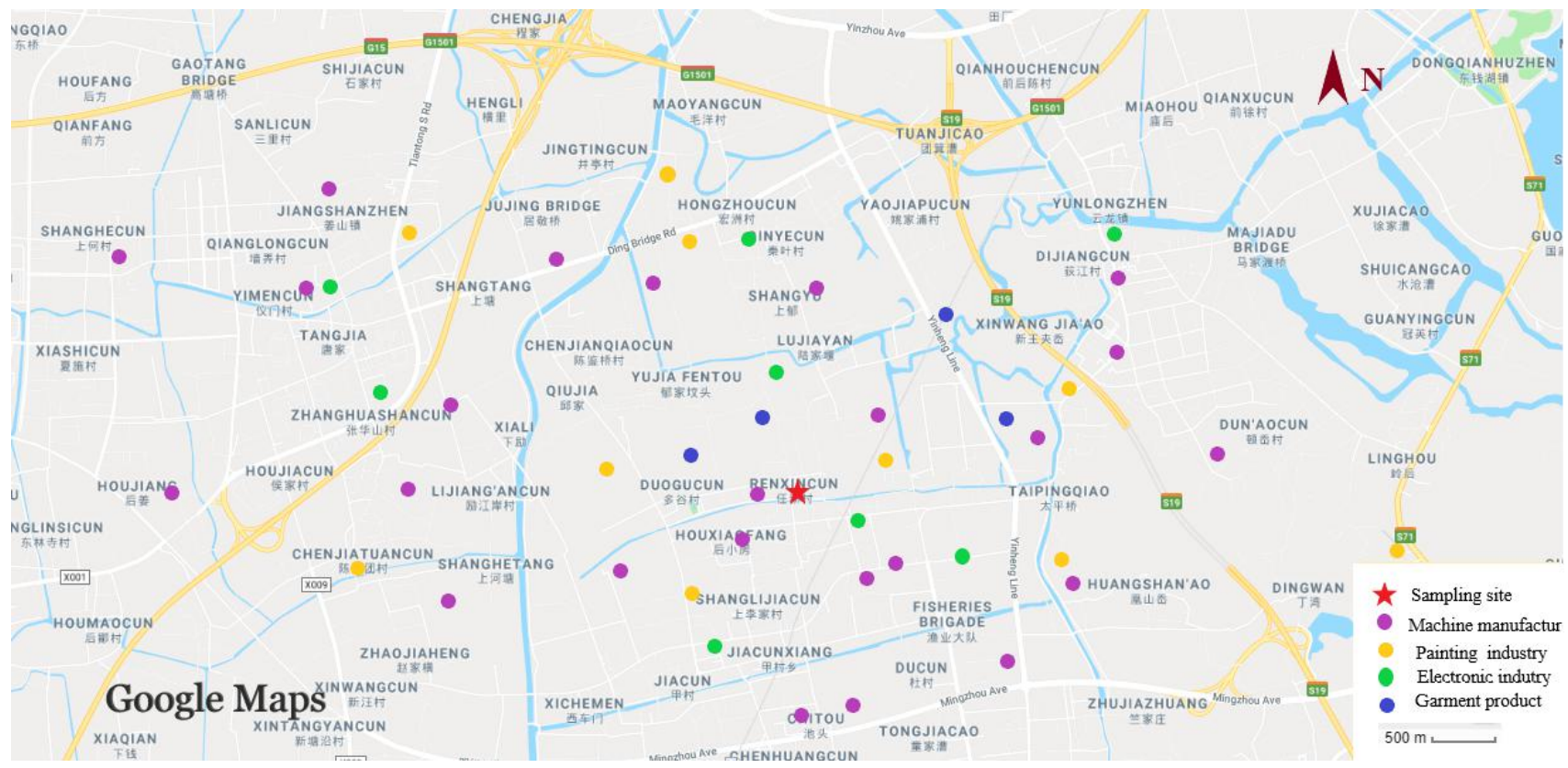

Figure S4. The spatial distributions of fluoride related products manufacturers in Zhejiang site (a small village in Ningbo City) 


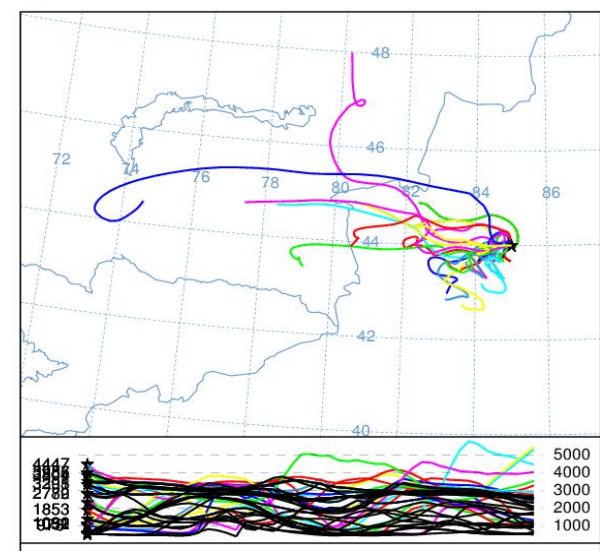

Tacheng, Xinjiang; Summer

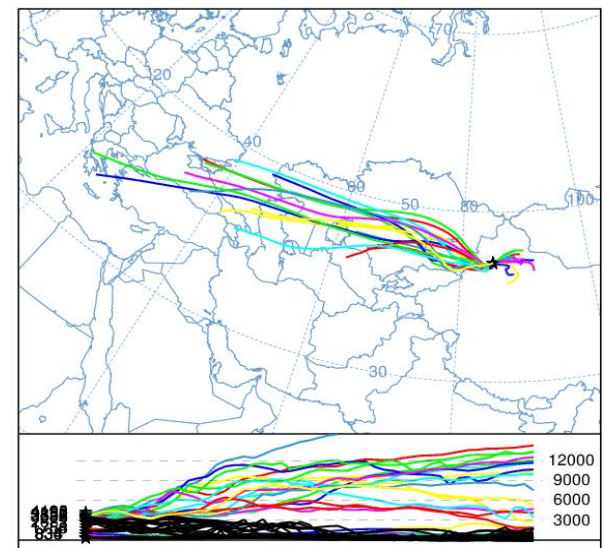

Tacheng, Xinjiang; Winter

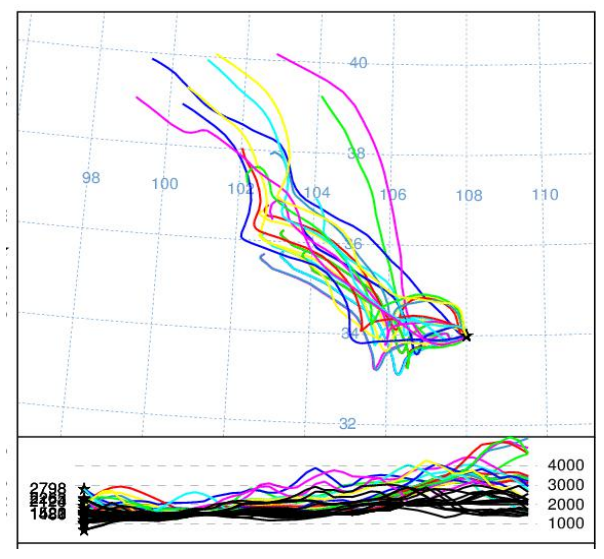

Xi'an, Shaanxi; Summer

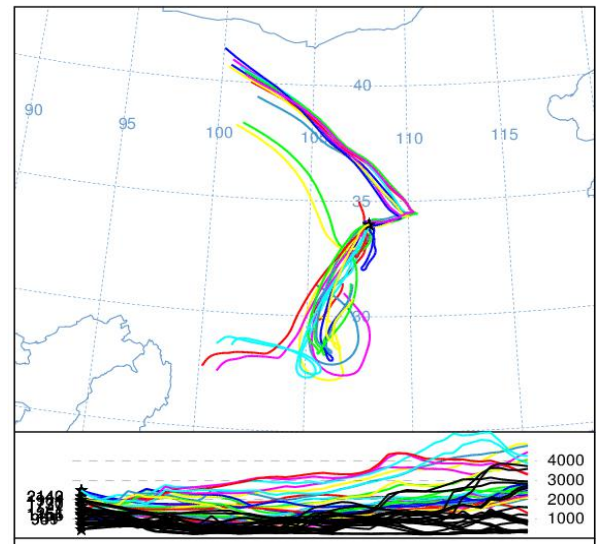

Xi'an, Shaanxi; Winter

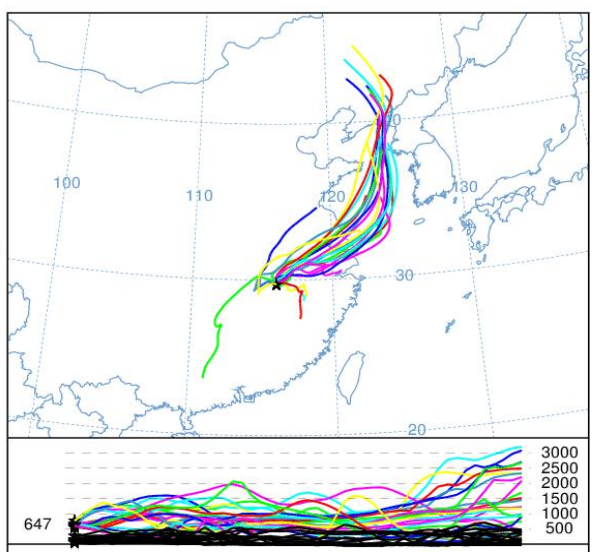

Jiujiang, Jiangxi; Summer

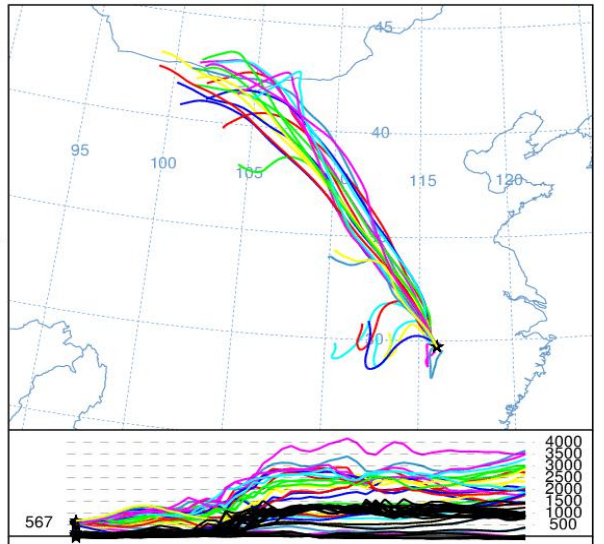

Jiujiang, Jiangxi; Winter

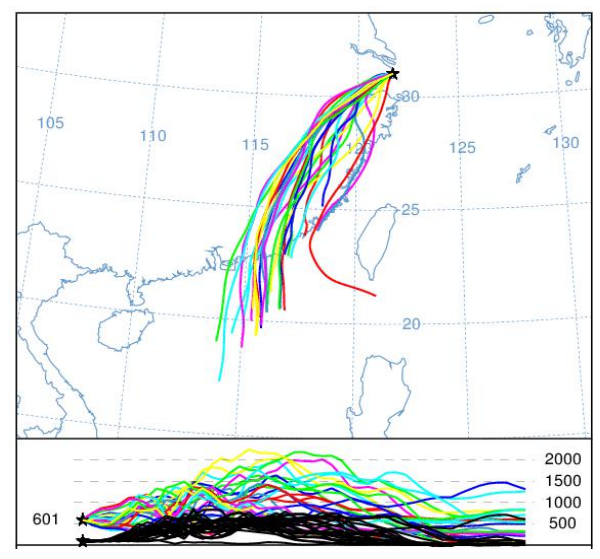

Shanghai; Summer

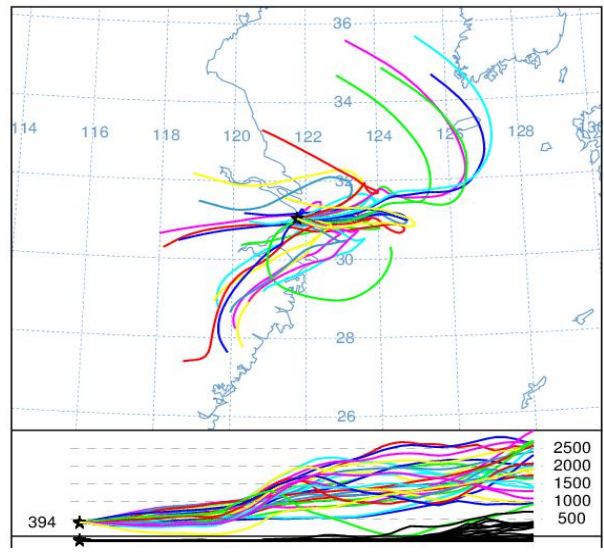

Shanghai; Winter 


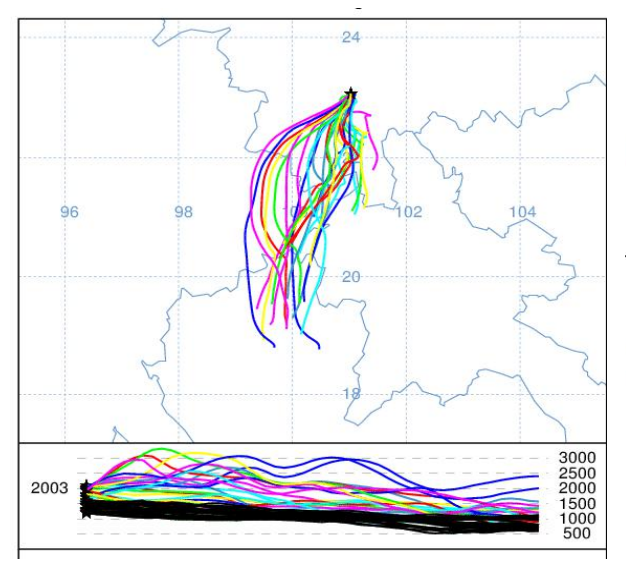

Pu'er, Yunnan; Summer

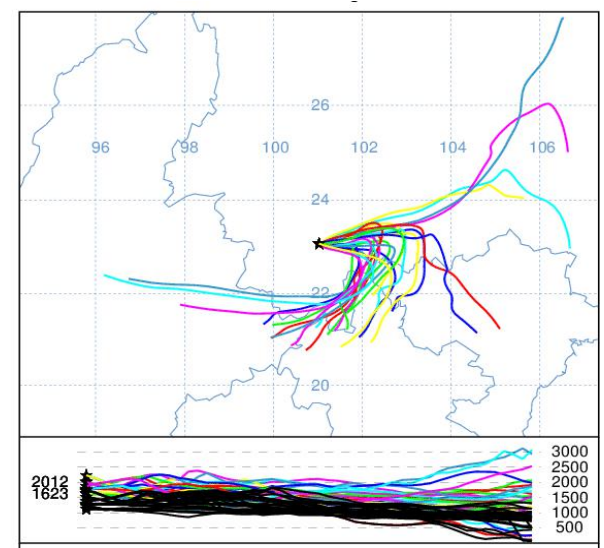

Pu'er, Yunnan; Winter

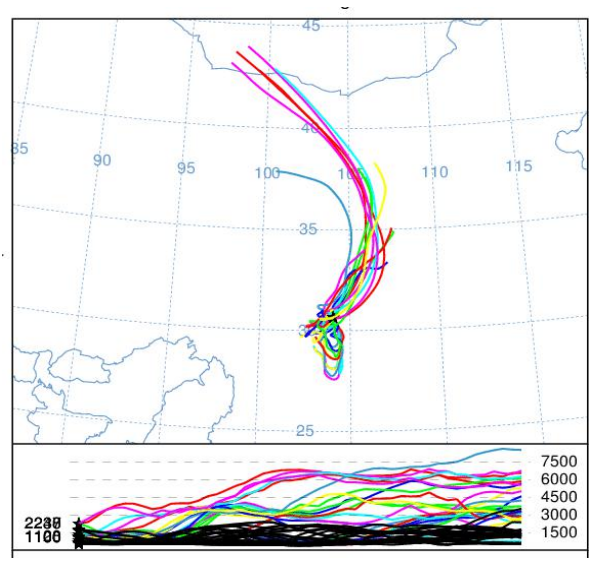

Chengdu, Sichuan; Summer

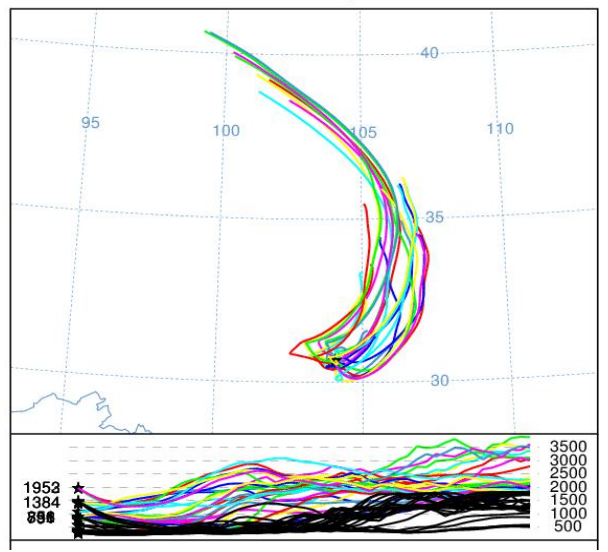

Chengdu, Sichuan; Winter

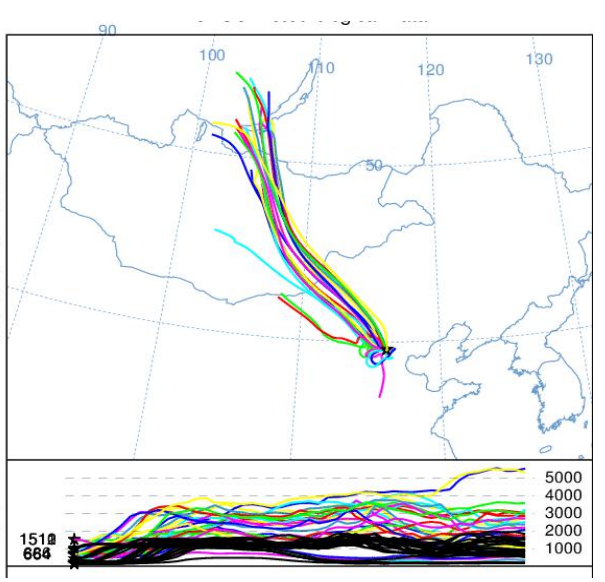

Beijing; Summer

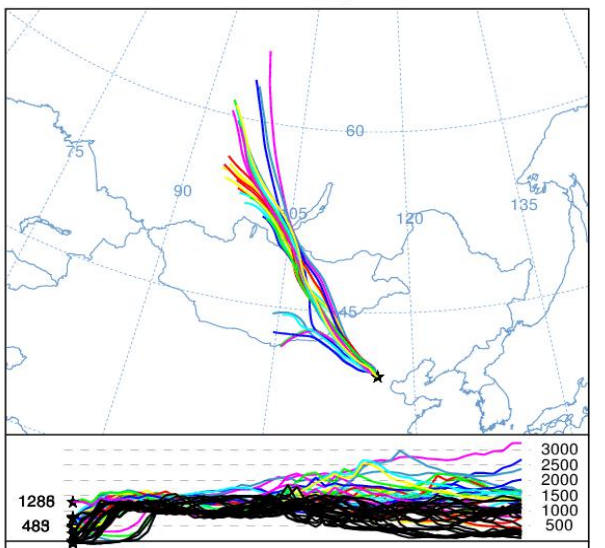

Beijing; Winter

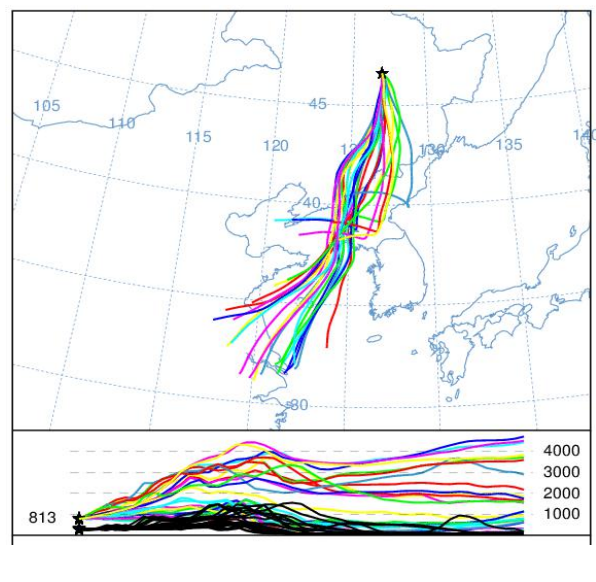

Suihua, Heilongjiang; Summer

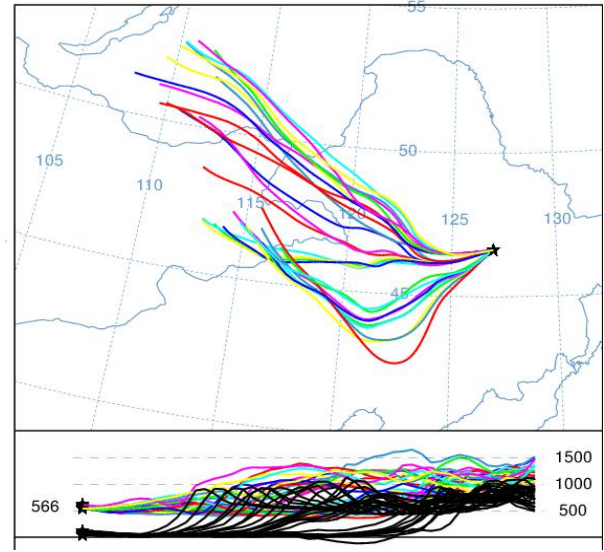

Suihua, Heilongjiang; Winter

Figure S5. The backward trajectories of air mass extracted by Hysplit trajectory model 


\section{Section S1. Sampling rate of XAD-PAS in this investigation}

Sampling rate of XAD-PAS is a crucial factor to derive air concentrations from the amounts of chemicals accumulated in the XAD resin. Previous literature suggested the sampling rate of XAD-PAS of 3.5-4.5 $\mathrm{m}^{3} / \mathrm{d}$ for PFASs (Li, Vento et al. 2011, Liu, Zhang et al. 2015, Tian, Yao et al. 2018). However, the actual sampling rate is dynamically variable, and affected by several factors. In this study, a standard solution containing mass labeled $1,2,3,4-{ }^{13} \mathrm{C}_{4}-\mathrm{PFOA}$ and $1,2,3,4-{ }^{13} \mathrm{C}_{4}-\mathrm{PFOS}(20 \mathrm{ng} / \mathrm{mL})$ was spiked directly onto the upper XAD resin in the Shanghai sampling site (Floor of 5-story building of School of Environmental Science and Engineering in Shanghai Jiao Tong University) for one month in April 2017, to account for analyte losses during sampling. The sampling rate was calculated as flowing formulas:

$$
\begin{aligned}
& \mathrm{R}=-\ln \left(\mathrm{C}_{\mathrm{t}} / \mathrm{C}_{0}\right) \times \mathrm{d} \times \mathrm{A} \times\left(K_{\mathrm{XAD}} / \mathrm{t}\right)=-\ln \left(\mathrm{C}_{\mathrm{t}} / \mathrm{C}_{0}\right) \times \mathrm{V} \times\left(K_{\mathrm{XAD}} / \mathrm{t}\right) \\
& \log K_{\mathrm{XAD}}=0.6366 \times \log \left(K_{\mathrm{OW}} \times \mathrm{S}_{\mathrm{W}} / \mathrm{S}_{\mathrm{A}}\right) \\
& \mathrm{S}_{\mathrm{A}}=\mathrm{P}_{\mathrm{L}} /(\mathrm{RT})
\end{aligned}
$$

where $\mathrm{C}_{t} / \mathrm{C}_{0}$ represents the measured recoveries of $1,2,3,4-{ }^{13} \mathrm{C}_{4}-\mathrm{PFOA}$ and $1,2,3,4-{ }^{13} \mathrm{C}_{4}-\mathrm{PFOS}$; $\mathrm{V}$ represents absorbent volume, $207.7\left(\mathrm{~cm}^{3}\right) ; K_{\mathrm{XAD}}$ represent ${ }^{13} \mathrm{C}_{8}-\mathrm{PFOA}$ partition coefficient between air and XAD; $\mathrm{t}$ represents sampling time, $30 \mathrm{~d}$; $K_{\mathrm{OW}}, \mathrm{S}_{\mathrm{W}}$, and $\mathrm{S}_{\mathrm{A}}$, represent octanol-air partition coefficient (6.3), water solubility, and air solubility, respectively; $P_{L}$ and $R$ represent liquid vapor pressure and gas constant $(8.314 \mathrm{~J} /(\mathrm{mol} \cdot \mathrm{K}))$, respectively. The $\log \mathrm{P}_{\mathrm{L}}$, and $\log \mathrm{S}_{\mathrm{W}}$ values was set as $1.3(\mathrm{~Pa})$, and $0.24(\mathrm{mg} / \mathrm{L})$ in the present study.

The sampling rate of XAD-PAS was calculated as $3.2 \mathrm{~m} 3 / \mathrm{d}$ in the selected geographical site. However, higher temperature and wind speed were suggested to have positive effect on sampler uptake efficiency, while negative effect on the sorption capacity. Although the sampling rate of PFAAs were proposed of site-specific under different meteorological conditions, we have not conduct the depuration compounds loss test in all the 23 sampling sites. Since our calculated XAD-PAS rate value was very close to the recommended rate of $3.5-4.5 \mathrm{~m}^{3} / \mathrm{d}$ for PFAAs, the rate value of $3.2 \mathrm{~m}^{3} / \mathrm{d}$ was used in the present study. 


\section{Section S2. PMF analysis and uncertainty assessment}

Positive matrix factorization (PMF) is considered an advanced algorithm among various receptor models, which has been successfully applied for source identification of environmental pollutants (Han, Fu et al. 2018; Han Fu et al. 2019). PMF has the following advantages: each data point is given an uncertainty-weighting; the factors in PMF are not necessarily orthogonal to each other and there is no non-negativity constraint with PMF. In the present study, PMF 5.0 (US EPA) was used to apportion the contributions of different sources to PFAAs in the atmosphere. The matrix X represents an ambient data set in which $\mathrm{i}$ represents the number of samples and $\mathrm{j}$ the number of chemical species. The goal of multivariate receptor modeling is to identify sources (p), the species profile (f) of each source and the amount of mass (g) contributed by each source to each individual sample as well as the residuals $\left(\mathrm{e}_{\mathrm{ij}}\right)$, as following equation:

$\mathrm{X}_{\mathrm{ij}}=\sum_{\mathrm{k}=1}^{\mathrm{p}} \mathrm{g}_{\mathrm{ik}} \mathrm{f}_{\mathrm{kj}}+\mathrm{e}_{\mathrm{ij}}$

The PMF solution minimizes the objective function Q based on these uncertainties $(u)$ :

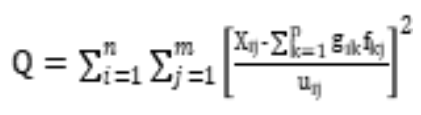

The input data files of PMF consist of concentrations and uncertainty matrices, and the uncertainty data were calculated as Equation (S3) as suggested by PMF User Guide. The missing values were represented by average values, while measurements below MDL (method detection limit) were replaced by two times of the corresponding MDL values. The “weak" variables were down-weighted, while "bad" variables were omitted form the analysis process.

$\begin{cases}\text { Unc }_{\mathrm{i}}=\frac{5}{6} \times \mathrm{MDL}_{\mathrm{i}} & \mathrm{C}_{\mathrm{i}} \leq \mathrm{MDL}_{\mathrm{i}} \\ \text { Unc }_{\mathrm{i}}=\sqrt{\left(\mathrm{C}_{\mathrm{i}} \times \text { Error Fraction }\right)^{2}+\left(\frac{1}{2} \times \mathrm{MDL}_{\mathrm{i}}\right)^{2}} & \mathrm{C}_{\mathrm{i}}>\mathrm{MDL}_{\mathrm{i}}\end{cases}$

The model was run 20 times with 49 random seeds to determine the stability of goodness-of-fit values.If the number of sources is estimated properly, the theoretical Q value should be approximately the number of degrees of freedom or the total number of data points. Three to six factors were examined, and four factors were found to be the most appropriate 
and most reasonably interpretable. Q (True) is the goodness-of-fit parameter calculated including all points, while Q (Robust) is the goodness-of-fit parameter calculated excluding points not fit by the model, Q (Robust) and Q (True) were 21672.9 and 25935 , respectively, with Q(true)/Qexp value of 12.56 . Additionally, approximately $97 \%$ of the residuals calculated by PMF were within the range of -3 to 3 , indicating a good fit of simulated results. The factor did not show oblique edges, suggesting there were little rotation for the solution. All these features implied the model simulation result was acceptable. 


\section{Reference}

Buck, R. C., et al. (2011). "Perfluoroalkyl and polyfluoroalkyl substances in the environment: terminology, classification, and origins." Integr Environ Assess Manag 7(4): 513-541.

Han, D., et al. (2018). "Non-polar organic compounds in autumn and winter aerosols in a typical city of eastern China: size distribution and impact of gas-particle partitioning on PM2.5 source apportionment." Atmos. Chem. Phys. 18(13): 9375-9391.

Han D , et al. (2019). "Investigate the impact of local iron-steel industrial emission on atmospheric mercury concentration in Yangtze River Delta, China." Environmental Science Pollution and Research. 26(6): $5862-5872$.

Karásková, P. et al. (2018). A critical assessment of passive air samplers for per- and polyfluoroalkyl substances, Atmos. Environ., 185, 186-195.

Li, J., et al. (2011). "Perfluorinated Compounds in the Asian Atmosphere." Environmental Science \& Technology 45(17): 7241.

Liu, B., et al. (2015). "Perfluorinated compounds (PFCs) in the atmosphere of Shenzhen, China: Spatial distribution, sources and health risk assessment." Chemosphere 138: 511-518.

Tian, Y., et al. (2018). "Occurrence and Phase Distribution of Neutral and Ionizable Per- and Polyfluoroalkyl Substances (PFASs) in the Atmosphere and Plant Leaves around Landfills: A Case Study in Tianjin,China." Environmental Science \& Technology 52(3): 1301.

Yu, S., et al. (2018). "Characteristics of perfluoroalkyl acids in atmospheric PM10 from the coastal cities of the Bohai and Yellow Seas, Northern China." Environmental Pollution 243: 1894-1903.

Hu, X. C., et al. (2016). "Detection of Poly- and Perfluoroalkyl Substances (PFASs)in U.S. Drinking Water Linked to Industrial Sites, Military Fire TrainingAreas, and Wastewater Treatment Plants." Environ Sci Technol Lett 3(10): 
344-350. 